\title{
The Involvement of Chronic Kidney Disease and Acute Kidney Injury in Disease Severity and Mortality in Patients with COVID-19: A Meta-Analysis
}

\author{
Bin Wang ${ }^{a, b}$ Qing Luo ${ }^{a}$ Weiguang Zhang ${ }^{b}$ Shuai $\mathrm{Yu}^{\mathrm{c}}$ Xiaowei Cheng ${ }^{\mathrm{a}}$ \\ Lanlan Wang ${ }^{a}$ Xiangmei Chen ${ }^{b}$ Yizhi Chen ${ }^{a, b}$ \\ ${ }^{a}$ Department of Nephrology, Hainan Hospital of Chinese PLA General Hospital, Hainan Academician Team \\ Innovation Center, Sanya, China; ${ }^{b}$ Department of Nephrology, The First Medical Center, Chinese PLA General \\ Hospital, Chinese PLA Institute of Nephrology, State Key Laboratory of Kidney Diseases, National Clinical Research \\ Center for Kidney Diseases, Beijing, China; ' Department of Tropical Medicine and Infectious Diseases, Hainan \\ Hospital of Chinese PLA General Hospital, Sanya, China
}

\section{Keywords}

COVID-19 - Chronic kidney disease - Acute kidney injury ·

Disease severity $\cdot$ Mortality $\cdot$ Meta-analysis

\section{Abstract}

Background: A meta-analysis was performed to evaluate the association of chronic kidney disease (CKD) and acute kidney injury (AKI) with the clinical prognosis of patients with coronavirus disease 2019 (COVID-19). Methods: The PubMed, EMBASE, Cochrane Library, medRxiv, Social Science Research Network, and Research Square databases (from December 1, 2019 to May 15, 2020) were searched to identify studies that reported the associations of CKD/AKI and disease severity/mortality. Pooled odds ratios (ORs) and 95\% confidence intervals (Cls) were calculated and meta-regression was performed. Results: In total, 42 studies enrolling 8,932 participants were included in this meta-analysis. The quality of most included studies was moderate to high. Compared with patients without previously diagnosed CKD, those with CKD had a significantly increased risk of progressing to a severe condition (OR 2.31,95\% Cl 1.64-3.24) or death (OR 5.11, 95\% Cl 3.36-7.77). Similarly, compared with pa-

karger@karger.com www.karger.com/kbr

Karger"

BOPEN ACCESS
(C) 2020 The Author(s)

Published by S. Karger AG, Basel

This article is licensed under the Creative Commons AttributionNonCommercial-NoDerivatives 4.0 International License (CC BY NC-ND) (http://www.karger.com/Services/OpenAccessLicense) Usage and distribution for commercial purposes as well as any distribution of modified material requires written permission. tients without $A K I$, those with $A K I$ had a significantly increased risk of progressing to a severe condition (OR 11.88, $95 \% \mathrm{Cl}$ 9.29-15.19) or death (OR 30.46, 95\% Cl 18.33-50.59). Compared with patients with previously diagnosed CKD, those with AKI were more likely to progress to a severe condition $\left(p_{\text {group }}<0.001, I^{2}=98.3 \%\right.$ ) and even to death $\left(p_{\text {group }}<\right.$ $\left.0.001, P^{2}=96.5 \%\right)$. Age had a significant impact on the association between CKD and disease severity $(p=0.001)$ but had no impact on the associations between AKI and disease severity $(p=0.80)$, between CKD and mortality $(p=0.51)$, or between AKI and mortality $(p=0.86)$. Four important complications (cardiac injury, shock, acute respiratory distress syndrome, and liver injury) did not significantly affect the associations between CKD/AKI and disease severity/mortality, indicating that CKD/AKI may be independent clinical prognostic indicators for patients with COVID-19. Conclusions: In COVID-19 patients, CKD/AKI was associated with worse outcomes compared with those without CKD/AKI. AKI was associated with higher risks of severity and mortality than CKD.

C) 2020 The Author(s)

Published by S. Karger AG, Basel

B. Wang and Q. Luo contributed equally to this work. 


\section{Introduction}

Since December 2019, a severe pneumonia outbreak caused by the severe acute respiratory syndrome coronavirus 2 (SARS-CoV-2) has spread rapidly around the world [1]. On February 11, 2020 the World Health Organization declared the name of the pandemic condition to be coronavirus disease 2019 (COVID-19).

In patients with severe COVID-19, the infection may rapidly progress to hypoxemia, septic shock, acute respiratory distress syndrome (ARDS), need for intensive care unit (ICU) care, and even death. Recently, several reports have revealed that comorbidities or other conditions can affect the clinical progression of patients with COVID-19 $[2,3]$. Several meta-analyses have demonstrated the impact of diabetes [4], cardiac injury [5, 6], and chronic obstructive pulmonary disease and smoking [7] on the clinical progression of patients with COVID- 19 .

A meta-analysis reported that the incidence of acute kidney injury (AKI) was estimated to be $3 \%$ in hospitalized patients with COVID-19, while this incidence was increased to $19 \%$ in patients admitted to an ICU [8]. Serum creatinine levels $\geq 133 \mu \mathrm{mol} / \mathrm{L}$ were reported to be associated with disease severity in a meta-analysis (three studies enrolling 979 patients) [9]. Another meta-analysis (three studies enrolling 944 patients) reported that AKI was associated with a higher risk of mortality [10]. However, the number of studies included in these published meta-analyses was relatively small. During the past half year, numerous new studies evaluating the association of chronic kidney disease (CKD)/AKI and disease severity/ mortality have been published. Therefore, a systematic review of the accumulated evidence with the aim of providing an up-to-date assessment of the association between kidney impairment (CKD/AKI) and clinical prognosis (disease severity/mortality) in patients with COVID-19 is important.

\section{Methods}

\section{Literature Search}

This meta-analysis was performed in accordance with the Meta-Analysis of Observational Studies in Epidemiology (MOOSE) method [11]. The MOOSE checklist is provided in online supplementary Table S1 (for all online suppl. material, see www.karger. com/doi/10.1159/000512211). The databases (MEDLINE, Embase, and Cochrane Library) were systematically searched for eligible published studies, with the medRxiv, Social Science Research Network, and Research Square websites searched for eligible unpublished studies from December 1, 2019 to May 15, 2020. The key words "COVID-19," "2019 novel coronavirus infection," "corona- virus disease 2019," "coronavirus," “SARS-CoV-2,” "2019-nCoV," "mortality," "severe," "survival," "outcomes," "prognosis," "chronic kidney disease," "acute renal failure," "acute kidney injury," and "renal replacement therapy" were used in various combinations.

\section{Study Eligibility Criteria}

After candidate articles had been collected, further selection was conducted according to the following inclusion criteria: (1) Adult patients. (2) The numbers of patients who were diagnosed with CKD or AKI were reported or could be calculated. In the absence of explicit definitions of CKD or AKI in the included studies, patients with high serum creatinine before or on admission were considered to be diagnosed with CKD (meeting the diagnostic criteria of the Kidney Disease: Improving Global Outcomes [KDIGO] guidelines) [12], while patients who had an increase in serum creatinine (meeting the diagnostic criteria of the KDIGO guidelines [12]) after SARS-CoV-2 infection were considered to have AKI. The definition of AKI was the same as that in the KDIGO guidelines. Patients with both preexisting CKD before infection and an increase in serum creatinine after infection were still considered to be diagnosed with CKD, but not AKI, during data extraction and synthesis. (3) The primary outcomes were disease severity and mortality. Diagnosis of the severe cases was defined by the authors in each individual study. Most of the included studies defined severe cases as ICU admission, mechanical ventilation, or both. In the absence of an explicit definition of severe cases, the guidelines for the diagnosis and treatment of SARS-CoV-2 issued by the National Health Commission of China (sixth edition) were used [13]. In detail, severe cases were defined as patients with dyspnea, respiratory rate $\geq 30 / \mathrm{min}$, blood oxygen saturation $\leq 93 \%$, partial pressure of arterial oxygen to fraction of inspired oxygen ratio $<300$, lung infiltrates $>50 \%$ within $24-48 \mathrm{~h}$, or needing ICU care. (4) The patients were to be consecutively confirmed and enrolled. The studies were in the following cases: (1) reviews, editorials, conference abstracts, systematic reviews, and meta-analyses; (2) children < 18 years old; (3) insufficient data provided to explore the associations between kidney impairment and the clinical outcomes; (4) repeated or updated reports containing or overlapping with the same group of participants.

\section{Data Extraction and Quality Assessment}

The extracted data included publication status, study type, regions/countries, enrollment hospitals and departments, enrollment periods, numbers of patients, age, sex, complications (cardiac injury, ARDS, shock, and liver injury), and treatment strategies. Quality assessment of the studies was conducted using the Newcastle-Ottawa Scale (NOS) for all included studies [14]. Eight different domains, including selection bias (adequate case definition, representativeness of the cases, selection of controls, and definition of controls), comparability (comparability of cases and controls on the basis of the design or analysis), and exposure (ascertainment of exposure, same method of ascertainment for cases and controls, and reports of nonresponse rate) were assessed. The total scores for each included study ranged from 0 to 10 points. These scores were chosen a priori to simplify description for the present review.

\section{Data Synthesis and Statistical Analysis}

Dichotomous variables were expressed as odds ratio (OR) and 95\% confidence interval (CI). Heterogeneity was assessed using
Wang/Luo/Zhang/Yu/Cheng/Wang/ Chen/Chen 


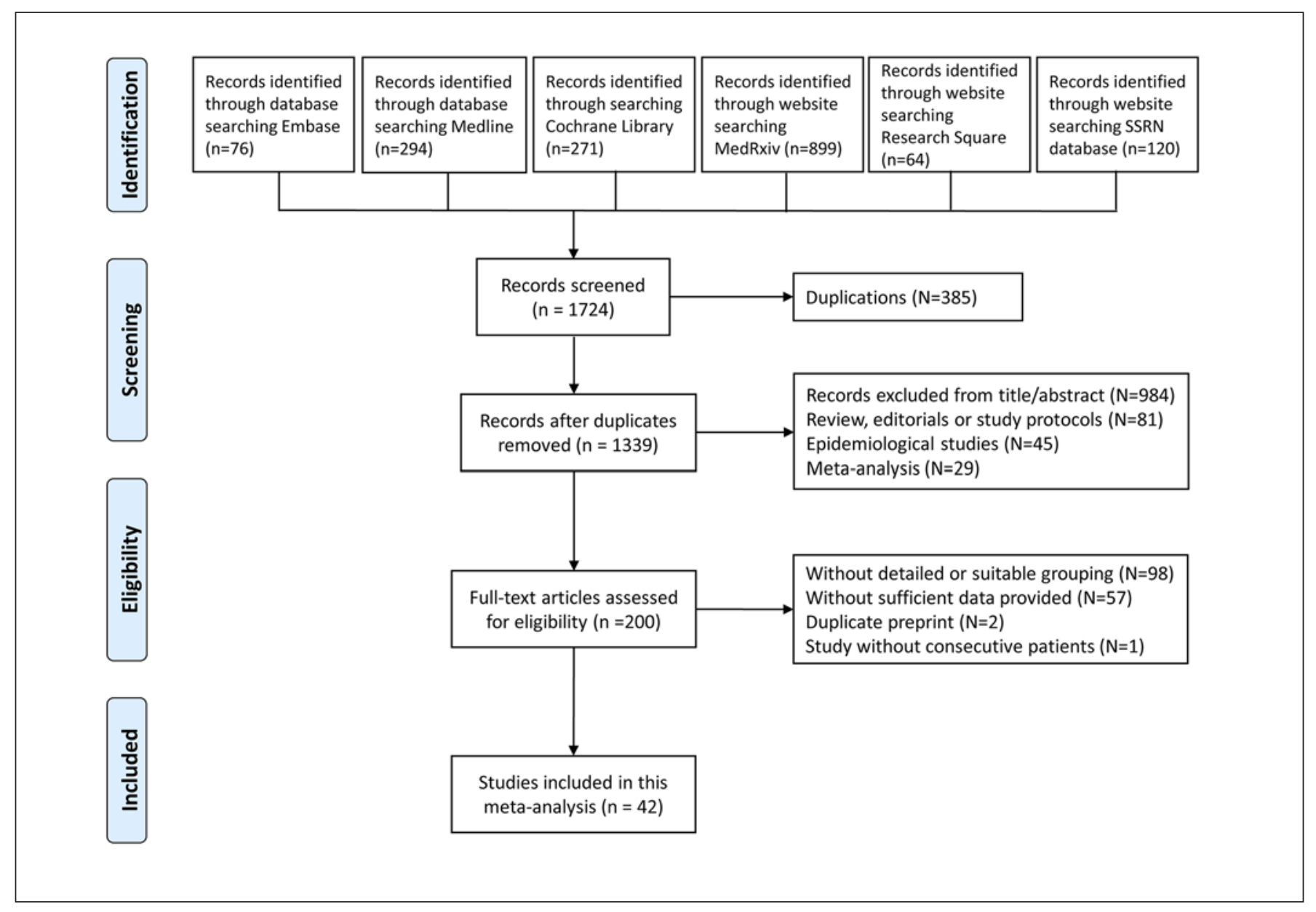

Fig. 1. Flow diagram of search strategy and study selection. SSRN, Social Science Research Network.

the Q test and quantified using the $I^{2}$ statistic [15]. The threshold $p$ value of heterogeneity was $0.10 . I^{2}$ statistics $<25 \%, 25-49 \%, 50-$ $75 \%$, and $>75 \%$ were interpreted to indicate low, medium, high, and very high levels of heterogeneity, respectively. If the $I^{2}$ value was $<50 \%$, the fixed-effect model was used. Otherwise, the random-effects model was used. For subgroup difference analysis, the $I^{2}$ value indicated the percentage of the variability in effect estimates from the different subgroups that is due to genuine subgroup differences rather than sampling error. Publication bias was explored using a funnel plot if more than 10 studies were included. Subgroup analyses were performed to evaluate whether the results differed according to the location of the studies (Wuhan city or non-Wuhan regions) and the publication status (published or unpublished). Meta-regression was performed to investigate the effects of age and complications such as cardiac injury, ARDS, shock, and liver injury on the relationship between kidney impairment and clinical prognosis. The Review Manager (version 5.3, The Cochrane Collaboration) software was used for data synthesis and publication bias. The STATA 14.0 (StataCorp, College Station, TX, USA) software was used for meta-regression. The searching and selection of the studies, data extraction and quality assessment, data synthesis, and statistical analysis were performed indepen-

Meta-Analysis of CKD/AKI with Severity/

Mortality in COVID-19 Patients dently by two of the researchers (B. Wang and Q. Luo), and any discrepancies were resolved by consulting a third investigator (Y. Chen).

\section{Results}

\section{Literature Search Results}

A total of 1,724 papers were screened, and finally 42 studies with 8,932 participants were included in this meta-analysis. Eighteen of the 42 studies were published [16-33], and the remaining 24 studies were rapidly posted on the medRxiv, Social Science Research Network, and Research Square websites without peer review [34-57]. A flow diagram outlining the literature search process is provided in Figure 1. The characteristics of the included studies are presented in Table 1 . The median number of participants was 147 (range 16-1,000). Twelve studies 


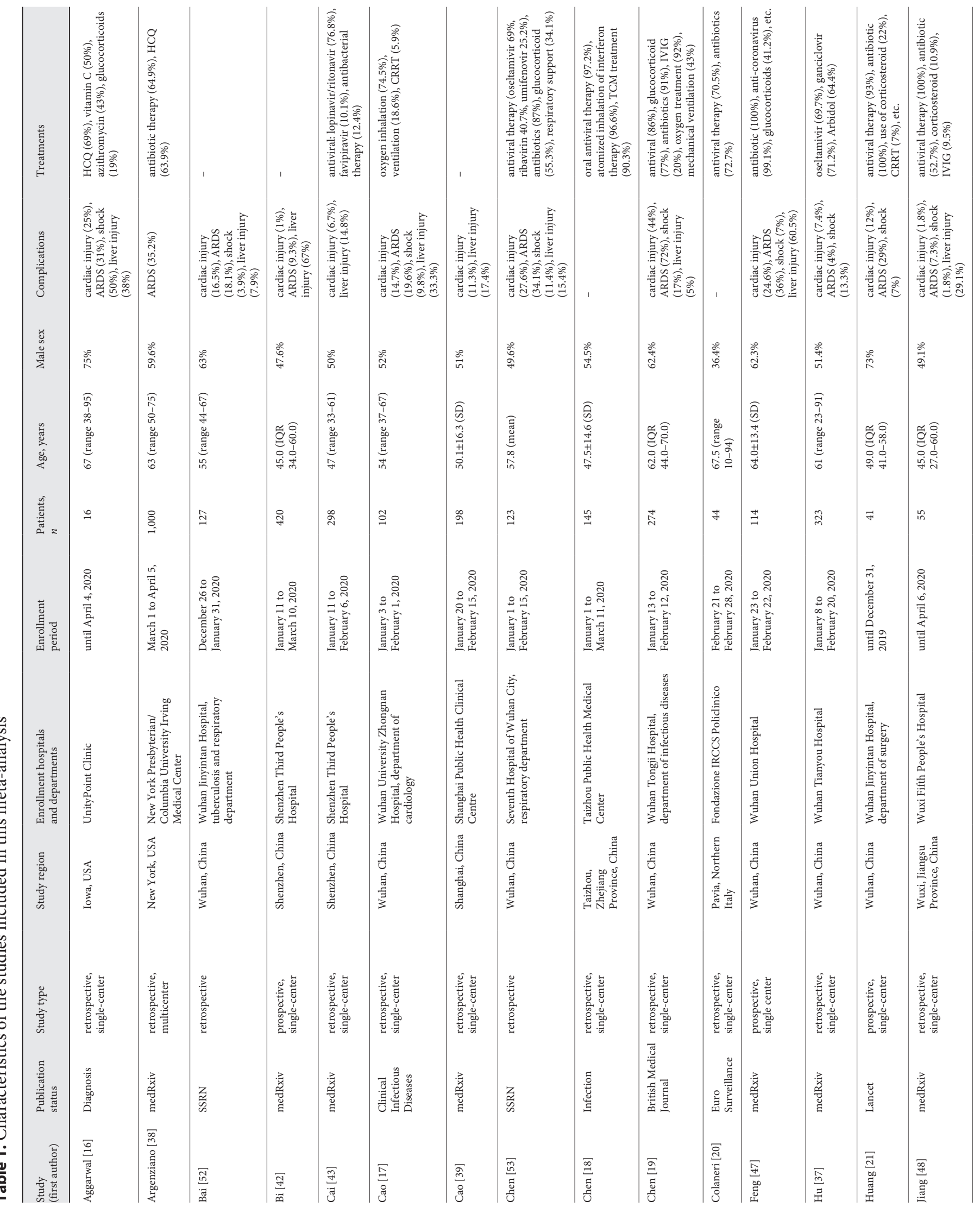




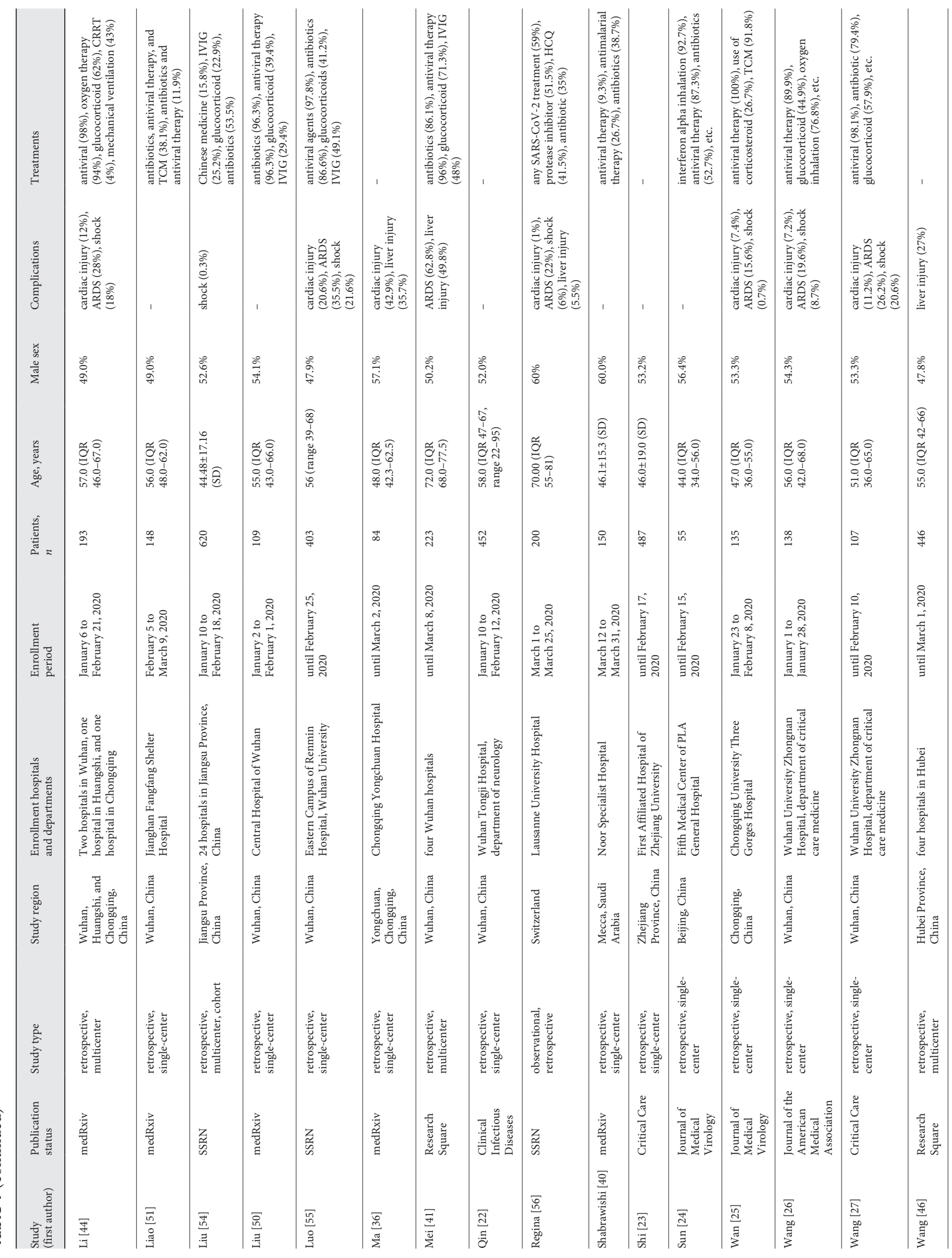




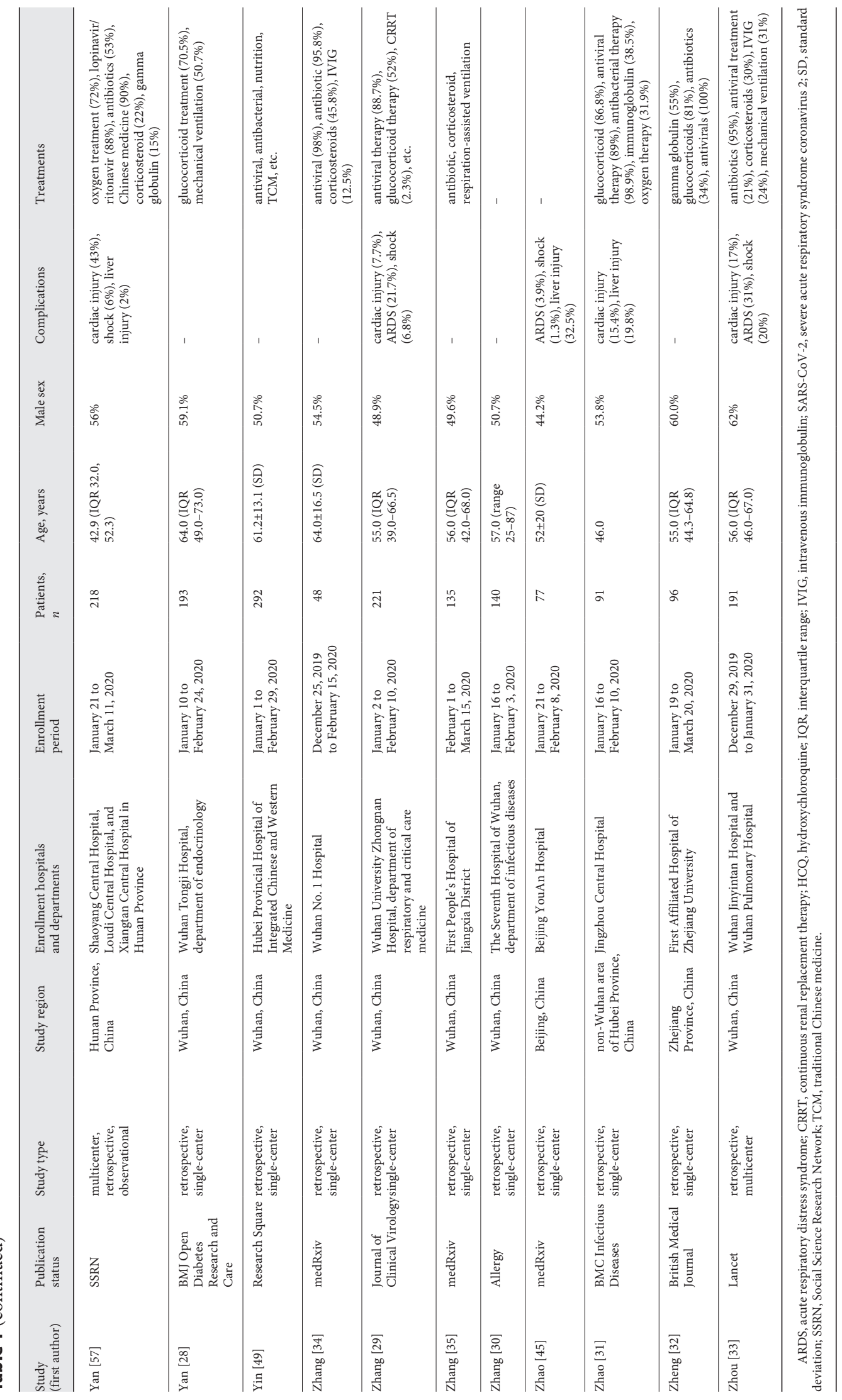




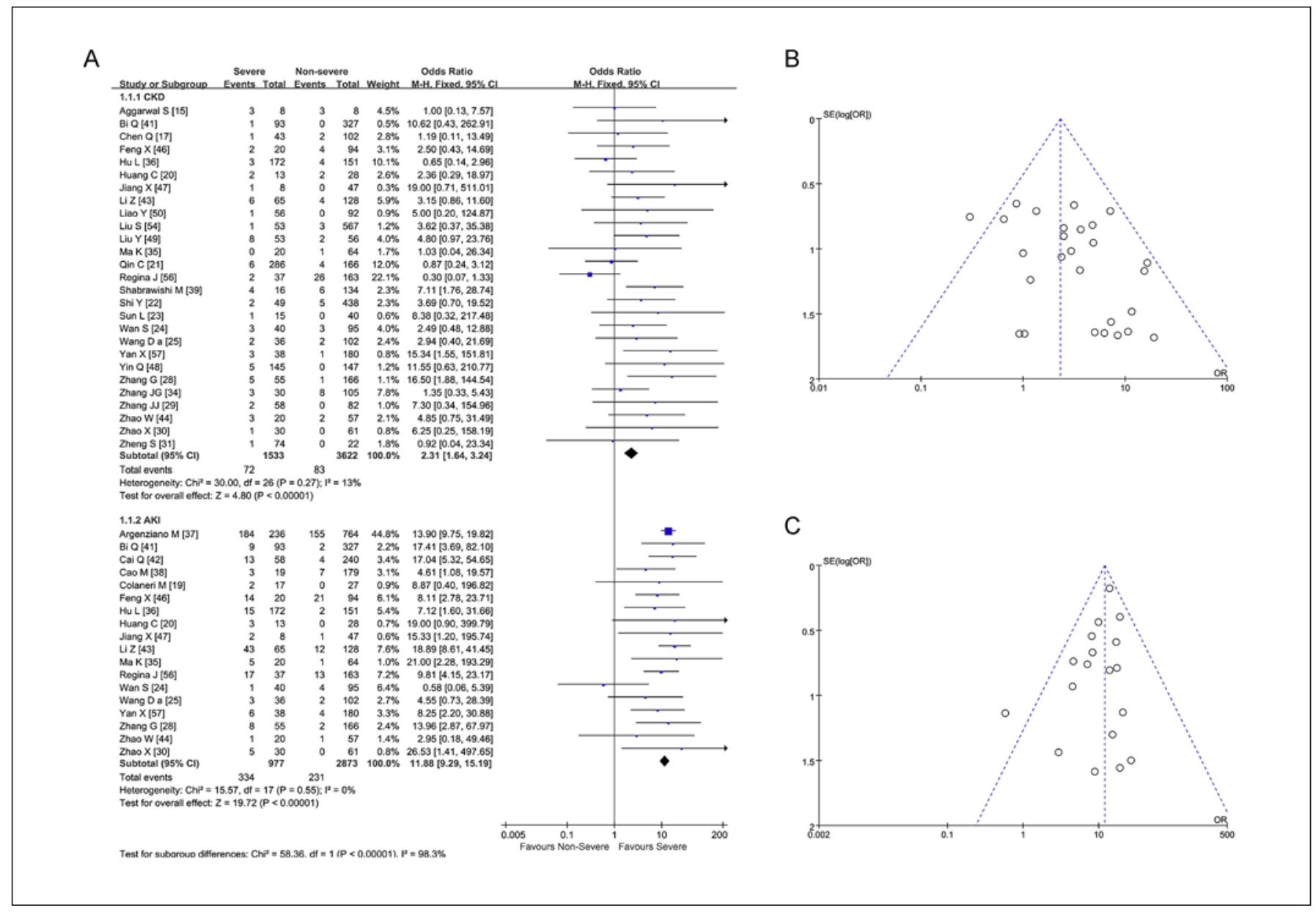

Fig. 2. Association between CKD/AKI and disease severity in patients with COVID-19. A Forest plot analyzing the association of CKD/AKI with disease severity in patients with COVID-19. B Funnel plot analyzing the publication bias in the association of CKD with disease severity. C Funnel plot analyzing the publication bias in the association of AKI with disease severity. AKI, acute kidney injury; CKD, chronic kidney disease; COVID-19, coronavirus disease 2019 . used mortality as the primary outcome, 29 studies used disease severity as the primary outcome, and 1 study used both disease severity and mortality as the primary outcomes. Five studies were performed outside China ( 2 in the United States [16, 38], 1 in Italy [20], 1 in Switzerland [56], and 1 in Saudi Arabia [40]). The remaining 37 studies were conducted in China (22 in Wuhan City and 15 in non-Wuhan regions). In the studies conducted in China and Saudi Arabia, severe disease was defined by the National Health Commission of China criteria [13]. In the Italian study, severe disease was defined as a requirement for high-flow oxygen support. In the study conducted in Switzerland, severe disease was defined as the requirement for mechanical ventilation. For the studies conducted in the United States, severe disease was defined as the need for mechanical ventilation or ICU admission. Twenty-two of the included studies were performed in Wuhan city. Among them, 2 studies were conducted in two different departments with different enrollment periods (surgery department until December 31 [21] and respiratory department from December 26 to January 31 [52]) in the same hospital (Wuhan Jinyintan Hospital). Two studies were conducted in two different departments with overlapping enrollment periods (respiratory department from January 1 to February 15 [53] and infectious disease department from January 16 to February 3 [30]) in the same hospital (Seventh Hospital of Wuhan City). Three studies were conducted in three different departments with similar enrollment periods (infectious disease department from January 13 to February 12 [19], neurology 
department from January 10 to February 12 [22], and endocrinology department from January 10 to February 24 [28]) in the same hospital (Wuhan Tongji Hospital). Four studies were conducted in three different departments with similar enrollment periods (ICU department from January 1 to January 28 [26], ICU department until February 10 [27], cardiology department from January 3 to February 1 [17], and respiratory department from January 2 to February 10 [29]) in the same hospital (Wuhan University Zhongnan Hospital). The remaining 11 studies were conducted in different hospitals.

\section{Quality Assessment Results}

Twenty-eight studies had NOS points ranging from 6 to 7 (13 studies scored 6 points and 15 studies scored 7 points), 14 studies had NOS points $>7$ ( 8 studies scored 8 points and 6 studies scored 9 points), and no included study had $<6$ points (online suppl. Table S2).

\section{The Association of Previously Diagnosed CKD or AKI} with Disease Severity

A total of 27 studies with 5,155 patients reported an association between previously diagnosed CKD and disease severity (Fig. 2A). The overall prevalence of CKD was $3.03 \%(155 / 5,115)$ in all included studies. Compared with COVID-19 patients without previously diagnosed $\mathrm{CKD}$, those with previously diagnosed CKD had a significantly increased risk of progressing to a severe condition $\left(\mathrm{OR}=2.31,95 \%\right.$ CI 1.64-3.24, $p<0.001, I^{2}=13 \%$, $\left.p_{\text {heterogeneity }}=0.27\right)$. Eighteen studies with 3,850 patients reported an association between $\mathrm{AKI}$ and disease severity (Fig. 2A). The overall incidence of AKI was $14.68 \%$ $(565 / 3,850)$ in all included studies. Compared with COVID-19 patients without AKI, those with AKI had a significantly increased risk of progressing to a severe condition $\left(\mathrm{OR}=11.88,95 \%\right.$ CI 9.29-15.19, $p<0.001, I^{2}=0 \%$, $\left.p_{\text {heterogeneity }}=0.55\right)$. Subgroup analysis indicated that patients with AKI were more likely to progress to a severe condition compared with patients with previously diagnosed CKD, which was demonstrated by the significant difference between the AKI and CKD groups (AKI vs. CKD: $\mathrm{OR}=11.88,95 \%$ CI 9.29-15.19 vs. OR $=2.31,95 \%$ CI 1.64-3.24; $p_{\text {group }}<0.001, I^{2}=98.3 \%$ ) (Fig. $2 \mathrm{~A}$ ). The funnel plots indicated no publication bias for the associations of CKD/AKI with disease severity (Fig. 2B, C).

Subgroup analyses indicated that the publication status did not significantly affect the associations between CKD/ AKI and disease severity (CKD: published vs. unpublished studies: $\mathrm{OR}=2.41,95 \% \mathrm{CI} 1.36-4.30$ vs. $\mathrm{OR}=2.25,95 \%$ CI 1.47-3.43, $p_{\text {subgroup }}=0.84, I^{2}=0 \%$; AKI: published vs. unpublished studies: $\mathrm{OR}=6.53,95 \%$ CI $3.02-14.11$ vs. $\mathrm{OR}=12.74,95 \%$ CI 9.82-16.52, $p_{\text {subgroup }}=0.11, I^{2}=$ $61.5 \%$ ) (online suppl. Fig. S1A, S1B). Similarly, subgroup analyses revealed that the geographic region did not significantly affect the associations of CKD/AKI with disease severity (CKD: Wuhan City vs. non-Wuhan regions: $\mathrm{OR}=$ $2.40,95 \%$ CI $1.43-4.01$ vs. $\mathrm{OR}=2.23,95 \%$ CI $1.41-3.51$, $p_{\text {subgroup }}=0.83, I^{2}=0 \%$; AKI: OR $=11.67,95 \%$ CI 6.90 19.73 vs. $\mathrm{OR}=11.95,95 \%$ CI $9.06-15.77, p_{\text {subgroup }}=0.94$, $I^{2}=0 \%$ ) (online suppl. Fig. S2A, S2B). Neither the publication status nor the geographic region had an influence on the associations of $\mathrm{CKD} / \mathrm{AKI}$ with disease severity.

\section{The Association of Previously Diagnosed CKD or AKI} with Disease Mortality

Eleven studies with 2,140 participants reported an association between previously diagnosed $\mathrm{CKD}$ and disease mortality (Fig. 3A). The overall prevalence of CKD was $6.73 \%(144 / 2,140)$ in all included studies. Compared with COVID-19 patients without previously diagnosed CKD, those with previously diagnosed CKD had a significantly increased risk of death $(\mathrm{OR}=5.11,95 \%$ CI 3.36-7.77, $p<$ $0.001, I^{2}=0 \%$, $p_{\text {heterogeneity }}=0.68$ ). Six studies with 1,220 patients reported an association between AKI and disease mortality. The incidence of AKI was $13.28 \%(162 / 1,220)$ in all included studies. Compared with COVID-19 patients without AKI, those with AKI had a significantly increased risk of death, with medium heterogeneity $\left(\mathrm{OR}=30.46,95 \%\right.$ CI $18.33-50.59, p<0.001, I^{2}=42 \%$, $p_{\mathrm{h}}$ eterogeneity $=0.12)($ Fig. 3A). Subgroup analysis indicated that patients with AKI were more likely to die than patients with previously diagnosed CKD, which was demonstrated by the significant difference between the AKI and $\mathrm{CKD}$ groups (AKI vs. CKD: $\mathrm{OR}=30.46,95 \% \mathrm{CI}$ $18.33-50.59$ vs. $\mathrm{OR}=5.11,95 \%$ CI $3.36-7.77, p_{\text {group }}<$ $0.001, I^{2}=96.5 \%$ ) (Fig. 3A). The funnel plot indicated no publication bias for the associations of CKD with disease mortality (Fig. 3B). Publication bias was not explored for the association of AKI and mortality because the number of included studies was $<10$.

Subgroup analysis indicated that the publication status did not significantly affect the association between CKD and disease mortality (published vs. unpublished studies: $\mathrm{OR}=4.77,95 \% \mathrm{CI} 1.93-11.77$ vs. $\mathrm{OR}=5.21,95 \%$ CI $3.25-$ $\left.8.36, p_{\text {subgroup }}=0.87, I^{2}=0 \%\right)$ (online suppl. Fig. S3A). However, subgroup analysis revealed that the publication status had a significant impact on the association of AKI with disease mortality (published vs. unpublished studies: $\mathrm{OR}=86.13,95 \%$ CI $25.26-293.68$ vs. $\mathrm{OR}=18.61,95 \%$ CI 10.49-33.02, $\left.p_{\text {subgroup }}=0.03, I^{2}=79.7 \%\right)$ (online suppl.
Wang/Luo/Zhang/Yu/Cheng/Wang/ Chen/Chen 
A

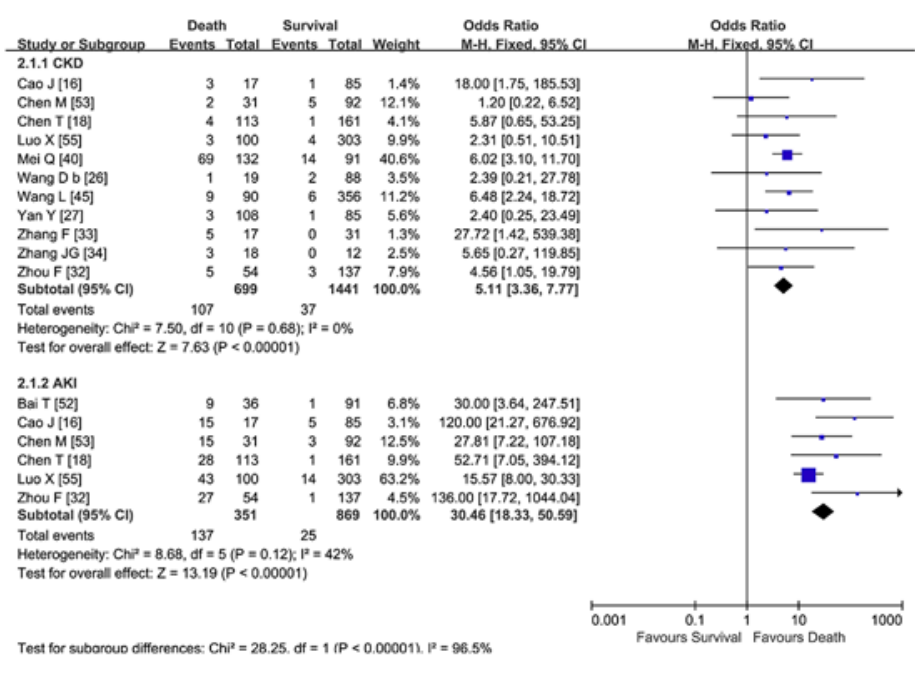

B

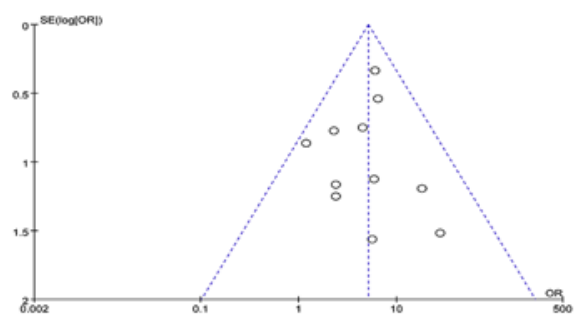

Fig. 3. Association between CKD/AKI and disease mortality in patients with COVID-19. A Forest plot analyzing the association of CKD/AKI with disease mortality in patients with COVID-19. B Funnel plot analyzing the publication bias in the association of $\mathrm{CKD}$ and disease mortality. AKI, acute kidney injury; CKD, chronic kidney disease; COVID-19, coronavirus disease 2019.

Table 2. Meta-regression analyzing the impact of complications on the association between kidney injury and clinical prognosis

\begin{tabular}{|c|c|c|c|c|c|c|c|c|}
\hline \multirow[t]{2}{*}{ Association } & \multicolumn{4}{|c|}{ Disease severity } & \multicolumn{4}{|c|}{ Disease mortality } \\
\hline & $\begin{array}{l}\text { cardiac } \\
\text { injury }\end{array}$ & ARDS & shock & $\begin{array}{l}\text { liver } \\
\text { injury }\end{array}$ & $\begin{array}{l}\text { cardiac } \\
\text { injury }\end{array}$ & ARDS & shock & $\begin{array}{l}\text { liver } \\
\text { injury }\end{array}$ \\
\hline CKD & $p=0.84$ & $p=0.51$ & $p=0.31$ & $p=0.43$ & $p=0.86$ & $p=0.55$ & $p=0.66$ & $p=0.33$ \\
\hline AKI & $p=0.93$ & $p=0.22$ & $p=0.07$ & $p=0.85$ & $p=0.82$ & $p=0.83$ & $p=0.14$ & $p=0.70$ \\
\hline
\end{tabular}

AKI, acute kidney injury; ARDS, acute respiratory distress syndrome; $\mathrm{CKD}$, chronic kidney disease.

Fig. S3B). The subgroup analyses to explore the impact of geographic region on the association of CKD/AKI with disease mortality were not performed because all the included studies were conducted in Wuhan City.

The Impact of Age and Complications on the

Association between Kidney Impairment and Clinical

Prognosis

Meta-regression analysis indicated that age had a significant impact on the association between $\mathrm{CKD}$ and disease severity ( $p=0.001$ ) (Fig. 4A). However, meta-regression analyses indicated that age did not significantly affect the associations between AKI and disease severity $(p=0.80)$ (Fig. 4B), between CKD and mortality ( $p=0.51$ ) (Fig. 4C), or between AKI and mortality ( $p=0.86$ ) (Fig. 4D). Metaregression analyses indicated that four important complications (cardiac injury, shock, ARDS, and liver injury) did not significantly affect the associations between CKD/AKI and disease severity/mortality (Table 2).

\section{Discussion}

We provide an up-to-date analysis of the evidence regarding the associations of CKD/AKI with clinical prognosis in patients with COVID-19 (42 studies with 8,932 patients). We demonstrated that COVID-19 patients with previously diagnosed CKD or AKI had significantly 


\section{A CKD and disease severity}

Meta-regression

REML estimate of between-study variance

\& residual variation due to heterogeneity

Proportion of between-study variance explained

with Knapp-Hartung modification

\begin{tabular}{r|rrrrrr}
\hline logor & $\exp (\mathrm{b})$ & Std. Err. & $t$ & $\mathrm{P}>|\mathrm{t}|$ & [95\% Conf. Interval] \\
\hline age & .9120811 & .0233848 & $\mathbf{- 3 . 5 9}$ & 0.001 & .8651687 & .9615373 \\
_cons & $\mathbf{3 7 7 . 6 9 8 2}$ & $\mathbf{5 3 3 . 4 0 3}$ & $\mathbf{4 . 2 0}$ & 0.000 & 20.60471 & $\mathbf{6 9 2 3 . 4 6 5}$ \\
\hline
\end{tabular}

Number of obs $=27$

tau2

I-squared res $=0.008$

\section{1]}

23.465

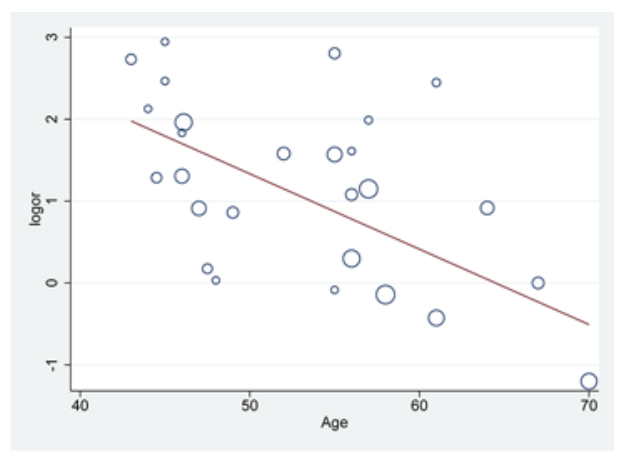

\section{B AKI and disease severity}

Meta-regression

REML estimate of between-study variance

\% residual variation due to heterogeneity

Proportion of between-study variance explained

with Knapp-Hartung modification

\begin{tabular}{r|rrrrrr}
\hline logor & $\exp (\mathrm{b})$ & Std. Err. & $t$ & $\mathrm{P}>|\mathrm{t}|$ & [95\% Conf. Interval] \\
\hline age & .9947018 & .0200643 & -0.26 & 0.796 & .9530638 & 1.038159 \\
_cons & 16.10508 & 18.18301 & 2.46 & 0.026 & 1.470661 & 176.3653 \\
\hline
\end{tabular}

Number of obs $=\quad 18$

tau2 $=.001753$

I-squared_res $=0.008$

Adj $\mathrm{R}$-squared $=.8$

西

.

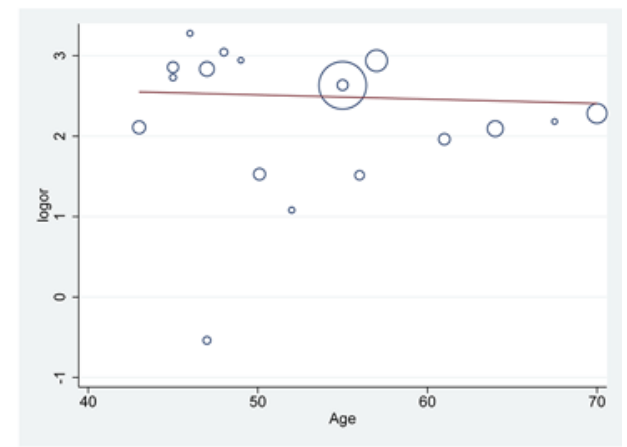

\section{CKD and disease mortality}

\begin{tabular}{|c|c|c|c|c|c|c|}
\hline \multicolumn{4}{|c|}{$\begin{array}{l}\text { Meta-regression } \\
\text { REML estimate of between-study variance } \\
\text { \% residual variation due to heterogeneity } \\
\text { Proportion of between-study variance explained } \\
\text { with Knapp-Hartung modification }\end{array}$} & & $\begin{array}{l}\text { Number of obs } \\
\text { tau2 } \\
\text { I-squared_res } \\
\text { Adj R-squared }\end{array}$ & $\begin{array}{rr}= & 11 \\
= & 0 \\
= & 0.008 \\
= & .8\end{array}$ \\
\hline logor & $\exp (b)$ & Std. Err. & t & $P>|t|$ & [95: Conf. & Interval] \\
\hline & 1.01935 & .0282674 & 0.69 & & .9573 & 1.085344 \\
\hline _cons & 1.48674 & 2.624723 & 0.22 & 0.827 & .0274037 & 80.66037 \\
\hline
\end{tabular}

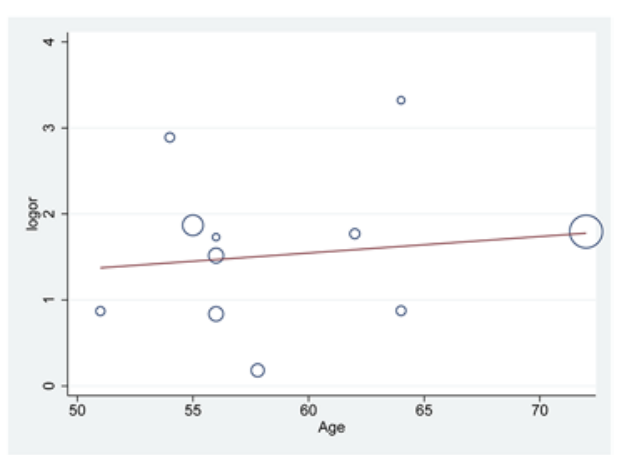

\section{AKI and disease mortality}

\begin{tabular}{|c|c|c|c|c|c|c|}
\hline \multicolumn{4}{|c|}{$\begin{array}{l}\text { Meta-regression } \\
\text { REML estimate of between-study variance } \\
\text { \& residual variation due to heterogeneity } \\
\text { Proportion of between-study variance explained } \\
\text { with Knapp-Hartung modification }\end{array}$} & & $\begin{array}{l}\text { Number of obs } \\
\text { tau2 } \\
\text { I-squared_res } \\
\text { Adj R-squared }\end{array}$ & $\begin{array}{lr}= & 6 \\
= & .5372 \\
= & 51.838 \\
= & -33.428\end{array}$ \\
\hline logor & $\exp (b)$ & Std. Err. & $t$ & $P>|t|$ & [95\% Conf. & Interval] \\
\hline & & .1852342 & -0.19 & & .5663989 & 1.644364 \\
\hline _cons & 287.9122 & 3134.406 & 0.52 & 0.630 & $2.15 e-11$ & $3.86 e+15$ \\
\hline
\end{tabular}

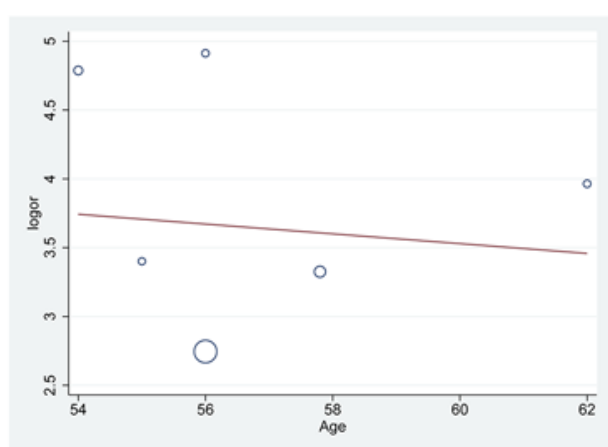

Fig. 4. Meta-regression investigating the impact of age on the association between $\mathrm{CKD} / \mathrm{AKI}$ and clinical prognosis. $\mathbf{A}$ Impact of age on the association between CKD and disease severity. B Impact of age on the association between AKI and disease severity. C Im- pact of age on the association between CKD and mortality. D Impact of age on the association between AKI and mortality. AKI, acute kidney injury; CKD, chronic kidney disease. 
increased risks of progression to a severe condition and even death. Compared with patients with previously diagnosed CKD before SARS-CoV-2 infection, patients with AKI after SARS-CoV-2 infection were more likely to progress to a severe condition or death.

Since the outbreak of the COVID-19 epidemic, several meta-analyses concerning kidney impairment and clinical prognosis have been published. $\mathrm{Ng}$ et al. [8] reported that the overall risk of AKI in hospitalized patients was 3\% and that this risk increased to $19 \%$ when patients were admitted to an ICU. Zheng et al. [9] found that serum creatinine could impact the risk of progression of COVID-19. Ali et al. [10] revealed that severe AKI was associated with a higher risk of mortality (relative risk $=3.08,95 \%$ CI 1.54-6.19). Potere et al. [58] reported that the incidence of AKI was 6\% in hospitalized patients. Lim et al. [59] demonstrated that AKI was associated with increased mortality, severe condition, and the need for ICU care. In our study, we demonstrated that not only previously diagnosed CKD but also AKI significantly affected the disease severity and mortality of COVID-19. We also found that AKI was associated with a more severe condition and a higher risk of mortality than CKD. Four major complications (cardiac injury, ARDS, shock, and liver injury) did not participate substantially in the associations between CKD/AKI and clinical prognoses, indicating that kidney impairment may be an independent clinical prognostic indicator for these patients.

The reason why COVID-19 patients with CKD comorbidity exhibited an increased risk of progression to a severe condition or death has not been fully elucidated to date. Plausible explanations are as follows: (1) Patients with CKD have a proinflammatory milieu and functional defects in innate and adaptive immune cell populations [60]. In a community-based cohort of nearly 10,000 adult individuals, reduced glomerular filtration rate and elevated albumin-creatinine ratios were associated with a higher risk of hospitalization, with infection and subsequent mortality [61]. (2) Patients with CKD have a high risk of upper respiratory tract infection and pneumonia $[62,63]$, which may become important concurrent infections with SARS-CoV-2. (3) CKD frequently coexists with comorbidities, especially diabetes and cardiovascular disease, which are also known to be associated with worse outcomes in patients with COVID-19 [9]. (4) CKD prevalence rises with age, and the burden of COVID-19 morbidity and mortality is heavily concentrated in older age groups. An important limitation of the present study is that we were unable to determine the extent to which age and comorbidities independently contribute to poor outcomes in patients with CKD.

Meta-Analysis of CKD/AKI with Severity/

Mortality in COVID-19 Patients
AKI is a syndrome of abrupt loss of kidney function that is strongly associated with increased mortality and morbidity in several conditions [64]. There is a high incidence of AKI in patients with COVID-19, especially in the cohort with severe disease $[46,49,65]$. The following reasons have been postulated to explain why an increased incidence of AKI occurs after SARS-CoV-2 infection: (1) The severity of the disease may be associated with an increase in the initial renal viral load or severe systemic inflammation, or both. SARS-CoV-2 can penetrate cells via two receptors - angiotensin-converting enzyme 2 (ACE2) and transmembrane protease, serine 2 (TMPRSS2) [66] -, and ACE2 is highly expressed in proximal tubular epithelial cells and in podocytes. Nine of 26 autopsied Chinese patients with AKI after SARS-CoV-2 infection had diffuse proximal tubular injury, with some frank necrosis and no glomerular injury [67]. (2) The fever, vomiting, diarrhea, and shock often observed with SARS-CoV-2 infection can cause kidney hypoperfusion. These reasons may cooperatively contribute to an increased risk of AKI. Additionally, COVID-19 patients with severe conditions also have complications involving various organ dysfunctions, which may in turn lead to AKI.

This meta-analysis has several limitations: (1) Twentytwo of the included studies were from Wuhan, China, and although it is unlikely that the same patients were included in multiple studies, the low heterogeneity in the outcome of our study may be attributable to the fact that these patients from the same region with similar genetic background were infected by the same strain of SARSCoV-2 virus in similar periods. This may limit generalizability, although subgroup analysis showed that the association with CKD/AKI and disease severity was consistent between studies from China and outside China. (2) Half of the included studies were posted on academic websites and were not peer-reviewed, and subgroup analysis demonstrated that the impact of AKI on the mortality in published studies was significantly higher than that in unpublished studies. This significant subgroup difference indicated the existence of publication bias. (3) The comparability of the baseline characteristics between the two groups (severe/nonsevere, survivors/deaths) was not well matched in a majority of studies, indicating that residual confounding is likely.

In conclusion, not only previously diagnosed CKD before SARS-CoV-2 infection, but also AKI after SARSCoV-2 infection were associated with disease severity and mortality. AKI had a higher risk of disease progression and death compared with CKD.

Kidney Blood Press Res 2021;46:17-30

DOI: $10.1159 / 000512211$ 


\section{Acknowledgment}

We would like to acknowledge Victoria Muir, $\mathrm{PhD}$, from Liwen Bianji, Edanz Group China (www.liwenbianji.cn/ac) for editing the English text of the manuscript.

\section{Statement of Ethics}

Our study adhered to the MOOSE guidelines [11]. Institutional approval and patient consent were not necessary.

\section{Conflict of Interest Statement}

The authors have no conflicts of interest to declare.

\section{Funding Sources}

This study was funded by the Specialized Scientific Program of the Hainan Province Academician Innovation Platform (YSPTZX202026).

\section{Author Contributions}

B. Wang, Q. Luo, Y. Chen, and Xiangmei Chen designed the study, with input into the study protocol from all authors. B. Wang, Q. Luo, and Y. Chen searched the literature and extracted the data. W. Zhang and S. Yu performed the statistical analyses. Xiaowei Cheng and L. Wang contributed to the discussion section. B. Wang and Q. Luo drafted the manuscript. Y. Chen and Xiangmei Chen supervised the study and provided critical revision to the intellectual content. All authors contributed to the interpretation of the data and approved the final version.

\section{References}

1 Calisher C, Carroll D, Colwell R, Corley RB, Daszak P, Drosten C, et al. Statement in support of the scientists, public health professionals, and medical professionals of China combatting COVID-19. Lancet. 2020 Mar; 395(10226):e42-3.

2 Renu K, Prasanna PL, Valsala Gopalakrishnan A. Coronaviruses pathogenesis, comorbidities and multi-organ damage - a review. Life Sci. 2020 Aug;255:117839.

3 Petrilli CM, Jones SA, Yang J, Rajagopalan H, O’Donnell L, Chernyak Y, et al. Factors associated with hospital admission and critical illness among 5279 people with coronavirus disease 2019 in New York City: prospective cohort study. BMJ. 2020 May;369:m1966.

4 Huang I, Lim MA, Pranata R. Diabetes mellitus is associated with increased mortality and severity of disease in COVID-19 pneumonia - a systematic review, meta-analysis, and meta-regression. Diabetes Metab Syndr. 2020 Jul-Aug;14(4):395-403.

5 Santoso A, Pranata R, Wibowo A, Al-Farabi MJ, Huang I, Antariksa B. Cardiac injury is associated with mortality and critically ill pneumonia in COVID-19: a meta-analysis. Am J Emerg Med. doi: 10.1016/j. ajem.2020.04.052 [Epub ahead of print].

6 Aggarwal G, Cheruiyot I, Aggarwal S, Wong J, Lippi G, Lavie CJ, et al. Association of Cardiovascular Disease With Coronavirus Disease 2019 (COVID-19) Severity: A MetaAnalysis. Curr Probl Cardiol. 2020 Aug;45(8): 100617.

7 Zhao Q, Meng M, Kumar R, Wu Y, Huang J, Lian N, et al. The impact of COPD and smoking history on the severity of COVID-19: A systemic review and meta-analysis. J Med Virol. 2020 Apr;92(10):1915-21.
8 Ng JJ, Luo Y, Phua K, Choong AM. Acute kidney injury in hospitalized patients with coronavirus disease 2019 (COVID-19): a metaanalysis. J Infect. 2020 Oct;81(4):647-79.

9 Zheng Z, Peng F, Xu B, Zhao J, Liu H, Peng J, et al. Risk factors of critical \& mortal COVID-19 cases: A systematic literature review and meta-analysis. J Infect. 2020 Aug; 81(2):e16-25.

10 Ali H, Daoud A, Mohamed MM, Salim SA, Yessayan L, Baharani J, et al. Survival rate in acute kidney injury superimposed COVID-19 patients: a systematic review and meta-analysis. Ren Fail. 2020 Nov;42(1):393-7.

11 Stroup DF, Berlin JA, Morton SC, Olkin I, Williamson GD, Rennie D, et al. Meta-analysis of observational studies in epidemiology: a proposal for reporting. Meta-analysis Of Observational Studies in Epidemiology (MOOSE) group. JAMA. 2000 Apr;283(15): 2008-12.

12 Levey AS, Eckardt KU, Dorman NM, Christiansen SL, Hoorn EJ, Ingelfinger JR, et al. Nomenclature for kidney function and disease: report of a Kidney Disease: Improving Global Outcomes (KDIGO) Consensus Conference. Kidney Int. 2020 Jun;97(6):1117-29.

13 National Health and Family Planning Commission of the People's Republic of China. Guideline for Diagnosis and Treatment of SARS-CoV-2 (6th edition). www.nhc.gov.cn/ yzygj/s7653p/202002/8334a8326dd94d329df 351d7da8aefc2.shtml [accessed February 19, 2020].

14 Stang A. Critical evaluation of the NewcastleOttawa scale for the assessment of the quality of nonrandomized studies in meta-analyses. Eur J Epidemiol. 2010 Sep;25(9):603-5.
15 Higgins JP, Green S. Cochrane Handbook for Systematic Reviews of Interventions Version 5.1.0 [updated March 2011]. The Cochrane Collaboration. https://handbook-5-1.cochrane.org [accessed May 15, 2020].

16 Aggarwal S, Garcia-Telles N, Aggarwal G, Lavie $C$, Lippi G, Henry BM. Clinical features, laboratory characteristics, and outcomes of patients hospitalized with coronavirus disease 2019 (COVID-19): early report from the United States. Diagnosis (Berl). 2020 May; 7(2):91-6.

17 Cao J, Tu WJ, Cheng W, Yu L, Liu YK, Hu X, et al. Clinical Features and Short-term Outcomes of 102 Patients with Coronavirus Disease 2019 in Wuhan, China. Clin Infect Dis. 2020 Jul;71(15):748-55.

18 Chen Q, Zheng Z, Zhang C, Zhang X, Wu H, Wang J, et al. Clinical characteristics of 145 patients with corona virus disease 2019 (COVID-19) in Taizhou, Zhejiang, China. Infection. 2020 Aug;48(4):543-51.

19 Chen T, Wu D, Chen H, Yan W, Yang D, Chen G, et al. Clinical characteristics of 113 deceased patients with coronavirus disease 2019: retrospective study. BMJ. 2020 Mar; 368:m1091.

20 Colaneri M, Sacchi P, Zuccaro V, Biscarini S, Sachs M, Roda S, et al.; COVID19 IRCCS San Matteo Pavia Task Force. Clinical characteristics of coronavirus disease (COVID-19) early findings from a teaching hospital in Pavia, North Italy, 21 to 28 February 2020. Euro Surveill. $2020 \mathrm{Apr} ; 25(16): 2000460$.

21 Huang C, Wang Y, Li X, Ren L, Zhao J, Hu Y, et al. Clinical features of patients infected with 2019 novel coronavirus in Wuhan, China. Lancet. 2020 Feb;395(10223):497-506. 
22 Qin C, Zhou L, Hu Z, Zhang S, Yang S, Tao Y, et al. Dysregulation of immune response in patients with coronavirus 2019 (COVID-19) in Wuhan, China. Clin Infect Dis. 2020 Jul; 71(15):762-8.

23 Shi Y, Yu X, Zhao H, Wang H, Zhao R, Sheng J. Host susceptibility to severe COVID-19 and establishment of a host risk score: findings of 487 cases outside Wuhan. Crit Care. 2020 Mar;24(1):108.

24 Sun L, Shen L, Fan J, Gu F, Hu M, An Y, et al. Clinical features of patients with coronavirus disease 2019 from a designated hospital in Beijing, China. J Med Virol. 2020 May;92(10): 2055-66.

25 Wan S, Xiang Y, Fang W, Zheng Y, Li B, Hu $\mathrm{Y}$, et al. Clinical features and treatment of COVID-19 patients in northeast Chongqing. J Med Virol. 2020 Jul;92(7):797-806.

26 Wang D, Hu B, Hu C, Zhu F, Liu X, Zhang J, et al. Clinical Characteristics of 138 Hospitalized Patients With 2019 Novel CoronavirusInfected Pneumonia in Wuhan, China. JAMA. 2020 Mar;323(11):1061-9.

27 Wang D, Yin Y, Hu C, Liu X, Zhang X, Zhou S, et al. Clinical course and outcome of 107 patients infected with the novel coronavirus, SARS-CoV-2, discharged from two hospitals in Wuhan, China. Crit Care. 2020 Apr;24(1): 188.

28 Yan Y, Yang Y, Wang F, Ren H, Zhang S, Shi $\mathrm{X}$, et al. Clinical characteristics and outcomes of patients with severe covid-19 with diabetes. BMJ Open Diabetes Res Care. 2020 Apr; 8(1):e001343.

29 Zhang G, Hu C, Luo L, Fang F, Chen Y, Li J, et al. Clinical features and short-term outcomes of 221 patients with COVID-19 in Wuhan, China. J Clin Virol. 2020 Jun;127:104364.

30 Zhang JJ, Dong X, Cao YY, Yuan YD, Yang YB, Yan YQ, et al. Clinical characteristics of $140 \mathrm{pa}$ tients infected with SARS-CoV-2 in Wuhan, China. Allergy. 2020 Jul;75(7):1730-41.

31 Zhao XY, Xu XX, Yin HS, Hu QM, Xiong T, Tang YY, et al. Clinical characteristics of patients with 2019 coronavirus disease in a nonWuhan area of Hubei Province, China: a retrospective study. BMC Infect Dis. 2020 Apr; 20(1):311.

32 Zheng S, Fan J, Yu F, Feng B, Lou B, Zou Q, et al. Viral load dynamics and disease severity in patients infected with SARS-CoV-2 in Zhejiang province, China, January-March 2020: retrospective cohort study. BMJ. 2020 Apr; 369:m1443.

33 Zhou F, Yu T, Du R, Fan G, Liu Y, Liu Z, et al. Clinical course and risk factors for mortality of adult inpatients with COVID-19 in Wuhan, China: a retrospective cohort study. Lancet. 2020 Mar;395(10229):1054-62.

34 Zhang F, Yang D, Li J, Gao P, Chen T, Cheng $Z$, et al. Myocardial injury is associated with in-hospital mortality of confirmed or suspected COVID-19 in Wuhan, China: a single center retrospective cohort study. Preprint at www.medrxiv.org/content/10.1101/2020.03. 21.20040121v1 [accessed May 15, 2020].
35 Zhang J, Ding D, Cao C, Zhang J, Huang X, $\mathrm{Fu}$ P, et al. Myocardial characteristics as the prognosis for COVID-19 patients. Preprint at www.medrxiv.org/content/10.1101/2020.05. 06.20068882v1 [accessed May 15, 2020]

36 Ma KL, Liu ZH, Cao CF, Liu MK, Liao J, Zou JB, et al. COVID-19 myocarditis and severity factors: an adult cohort study. Preprint at www.medrxiv.org/content/10.1101/2020.03. 19.20034124v1 [accessed May 15, 2020].

$37 \mathrm{Hu}$ L, Chen S, Fu Y, Gao Z, Long H, Ren HW, et al. Risk factors associated with clinical outcomes in 323 COVID-19 patients in Wuhan, China. Preprint at www.medrxiv.org/content $/ 10.1101 / 2020.03 .25 .20037721 \mathrm{v} 2$ [accessed May 15, 2020]

38 Argenziano MG, Bruce SL, Slater CL, Tiao JR, Baldwin MR, Barr RG, et al. Characterization and clinical course of 1000 Patients with COVID-19 in New York: retrospective case series. Preprint at www.medrxiv.org/content/1 0.1101/2020.04.20.20072116v2 [accessed May 15, 2020].

39 Cao M, Wang D, Wang Y, Lu Y, Zhu X, Li Y, et al. Clinical features of patients Infected with the 2019 novel coronavirus (COVID-19) in Shanghai, China. Preprint at www.medrxiv. org/content/10.1101/2020.03.04.20030395v1 [accessed May 15, 2020].

40 Shabrawishi M, Al-Gethamy MM, Naser AY, Ghazawi MA, Alsharif GF, Obaid EF, et al. Clinical, radiological and therapeutic characteristics of patients with COVID-19 in Saudi Arabia. Preprint at www.medrxiv.org/conten $\mathrm{t} / 10.1101 / 2020.05 .07 .20094169 \mathrm{v} 2$ [accessed May 15, 2020].

41 Mei Q, Wang AY, Yang Y, Li M, Wang F, Du $S$, et al. Survival factors and metabolic pathogenesis in elderly patients $(\geq 65)$ with COVID-19: a multi-center study of 223 cases. Preprint at www.researchsquare.com/article/rs23199/v1 [accessed May 15, 2020].

42 Bi Q, Hong C, Meng J, Wu Z, Zhou P, Ye C, et al. Characterizing clinical progression of COVID-19 among patients in Shenzhen, China: an observational cohort study. Preprint at www.medrxiv.org/content/10.1101/2020.04. 22.20076190v2 [accessed May 15, 2020]

43 Cai Q, Huang D, Ou P, Yu H, Zhu Z, Xia Z, et al. COVID-19 in a designated infectious diseases hospital outside Hubei Province, China. Allergy. 2020 Jul;75(7):1742-52.

44 Li Z, Wu M, Yao J, Guo J, Liao X, et al. Caution on kidney dysfunctions of COVID-19 patients. Preprint at www.medrxiv.org/conte nt/10.1101/2020.02.08.20021212v2 [accessed May 15, 2020].

45 Zhao W, Yu S, Zha X, Wang N, Pang Q, Li T, et al. Clinical characteristics and durations of hospitalized patients with COVID-19 in Beijing: a retrospective cohort study. Preprint at www.medrxiv.org/content/10.1101/2020.03. 13.20035436v2 [accessed May 15, 2020].
46 Wang L, Cheng X, Dong Q, Zhou C, Wang Y, Song B, et al. The characteristics of laboratory tests at admission and the risk factors for adverse clinical outcomes of severe and critical COVID-19. Preprint at www.researchsquare. com/article/rs-24018/v1 [accessed May 15, 2020].

47 Feng X, Li P, Ma L, Liang H, Lei J, Li W, et al. Clinical characteristics and short-term outcomes of severe patients with COVID-19 in Wuhan, China. Preprint at www.medrxiv. org/content/10.1101/2020.04.24.20078063v1 [accessed May 15, 2020].

48 Jiang X, Tao J, Wu H, Wang Y, Zhao W, Zhou $\mathrm{M}$, et al. Clinical features and management of severe COVID-19: a retrospective study in Wuxi, Jiangsu Province, China. Preprint at www.medrxiv.org/content/10.1101/2020.04 10.20060335v1 [accessed May 15, 2020].

49 Yin Q, Fu Z, Xie J, Yang J, Li F, Zhu W, et al. Analysis of risk factors of severe COVID-19 patients. Preprint at www.researchsquare. com/article/rs-23272/v1 [accessed May 15, 2020].

50 Liu Y, Sun W, Li J, Chen L, Wang Y, Zhang L, et al. Clinical features and progression of acute respiratory distress syndrome in coronavirus disease 2019. Preprint at www.medrxiv.org/content/10.1101/2020.02.17.20024 $166 \mathrm{v} 3$ [accessed May 15, 2020].

51 Liao Y, Feng Y, Wang B, Wang H, Huang J, $\mathrm{Wu} \mathrm{Y}$, et al. Clinical characteristics and risk factors for developed COVID-19 patients transferring to designated hospital from Jianghan Fangcang shelter hospital: a retrospective, observational study. Preprint at www.medrxiv.org/content/10.1101/2020.04. 21.20074724v1 [accessed May 15, 2020].

52 Bai T, Tu S, Wei Y, Xiao L, Jin Y, Zhang L, et al. Clinical and laboratory factors predicting the prognosis of patients with COVID-19: an analysis of 127 patients in Wuhan, China. Preprint at https://papers.ssrn.com/sol3/papers.cfm?abstract_id=3546118 [accessed May 15, 2020].

53 Chen M, Fan Y, Wu X, Zhang L, Guo T, Deng $\mathrm{K}$, et al. Clinical characteristics and risk factors for fatal outcome in patients with 2019-coronavirus infected disease (COVID-19) in Wuhan, China. Preprint at https:// papers.ssrn.com/sol3/papers.cfm?abstract id=3546069 [accessed May 15, 2020].

54 Liu S, Luo H, Wang Y, Wang D, Ju S, Yang Y. Characteristics and associations with severity in COVID-19 patients: a multicentre cohort study from Jiangsu province, China. Preprint at https://papers.ssrn.com/sol3/papers. cfm?abstract_id=3548753 [accessed May 15, 2020].

55 Luo X, Zhou W, Xia H, Yang W, Yan X, Wang $\mathrm{B}$, et al. Characteristics of SARS-CoV-2 infected patients with clinical outcome during epidemic ongoing outbreak in Wuhan, China. Preprint at https://papers.ssrn.com/sol3/ papers.cfm?abstract_id=3552812 [accessed May 15, 2020]. 
56 Regina J, Papadimitriou-Olivgeris M, Burger R, Filippidis P, Tschopp J, Desgranges F, et al. Epidemiology, risk factors and clinical course of SARS-CoV-2 infected patients in a Swiss university hospital: an observational retrospective study. Preprint at https://papers.ssrn. com/sol3/papers.cfm?abstract_id $=3578783$ [accessed May 15, 2020].

57 Yan X, Wang C, Peng D, Han X, Fan Y, Fang $\mathrm{Z}$, et al. Clinical features, treatment and outcomes of 218 patients with COVID-19: a retrospective, multicenter study based on clinical classification. Preprint at https://papers. ssrn.com/sol3/papers.cfm?abstract_ id $=3559594$ [accessed May 15, 2020].

58 Potere N, Valeriani E, Candeloro M, Tana M, Porreca E, Abbate A, et al. Acute complications and mortality in hospitalized patients with coronavirus disease 2019: a systematic review and meta-analysis. Crit Care. 2020 Jul; 24(1):389.
59 Lim MA, Pranata R, Huang I, Yonas E, Soeroto AY, Supriyadi R. Multiorgan failure with emphasis on acute kidney injury and severity of COVID-19: systematic review and meta-analysis. Can J Kidney Health Dis. 2020 Jul;7:2054358120938573.

60 Betjes MG. Immune cell dysfunction and inflammation in end-stage renal disease. Nat Rev Nephrol. 2013 May;9(5):255-65.

61 Ishigami J, Grams ME, Chang AR, Carrero JJ, Coresh J, Matsushita K. CKD and Risk for Hospitalization With Infection: The Atherosclerosis Risk in Communities (ARIC) Study. Am J Kidney Dis. 2017 Jun;69(6):752-61.

62 Cohen-Hagai K, Rozenberg I, Korzets Z, Zitman-Gal T, Einbinder Y, Benchetrit S. Upper Respiratory Tract Infection among Dialysis Patients. Isr Med Assoc J. 2016 Sep;18(9): $557-60$.
63 Sibbel S, Sato R, Hunt A, Turenne W, Brunelli SM. The clinical and economic burden of pneumonia in patients enrolled in Medicare receiving dialysis: a retrospective, observational cohort study. BMC Nephrol. 2016 Dec; 17(1):199.

64 Vanmassenhove J, Kielstein J, Jörres A, Biesen WV. Management of patients at risk of acute kidney injury. Lancet. 2017 May;389(10084): 2139-51.

65 Cheng Y, Luo R, Wang K, Zhang M, Wang Z, Dong L, et al. Kidney disease is associated with in-hospital death of patients with COVID-19. Kidney Int. 2020 May;97(5):829-38.

66 Wrapp D, Wang N, Corbett KS, Goldsmith JA, Hsieh CL, Abiona O, et al. Cryo-EM structure of the 2019-nCoV spike in the prefusion conformation. Science. 2020 Mar;367(6483): 1260-3.

67 Su H, Yang M, Wan C, Yi LX, Tang F, Zhu $\mathrm{HY}$, et al. Renal histopathological analysis of 26 postmortem findings of patients with $\mathrm{CO}$ VID-19 in China. Kidney Int. 2020 Jul;98(1): 219-27. 